BMJ Open

Diabetes

Research

\& Care

\section{Maximum body mass index before onset of type 2 diabetes is independently associated with advanced diabetic complications}

To cite: Ozawa H, Fukui K, Komukai S, et al. Maximum body mass index before onset of type 2 diabetes is independently associated with advanced diabetic complications. BMJ Open Diab Res Care 2021;9:e002466. doi:10.1136/ bmjdrc-2021-002466

Received 28 June 2021 Accepted 23 November 2021

\section{Check for updates}

(c) Author(s) (or their employer(s)) 2021. Re-use permitted under CC BY-NC. No commercial re-use. See rights and permissions. Published by BMJ.

${ }^{1}$ Department of Metabolic Medicine, Osaka University Graduate School of Medicine, Suita, Osaka, Japan 2Department of Integrated Medicine Division of Biomedical Statistics, Osaka University Graduate School of Medicine, Suita, Osaka, Japan

${ }^{3}$ Department of Community Medicine, Osaka University Graduate School of Medicine, Suita, Osaka, Japan

${ }^{4}$ Diabetes Center, Toyonaka Municipal Hospital, Toyonaka, Osaka, Japan

${ }^{5}$ Department of Diabetes Care Medicine, Osaka University Graduate School of Medicine, Suita, Osaka, Japan

Correspondence to Dr Kenji Fukui; kfukui@endmet.med.osaka-u. ac.jp

\section{ABSTRACT}

Introduction The maximum body mass index (BMI) before onset of type 2 diabetes (MBB0) might be used to estimate a patient's insulin secretion capacity. There have been few factors that can predict future diabetic complications at the time of diagnosis of diabetes mellitus. This study aimed to clarify the clinical usefulness of MBBO for predicting the development of advanced diabetic microvascular complications

Research design and methods This was a crosssectional observational study. Of 1304 consecutively admitted patients with type 2 diabetes, we enrolled 435 patients for whom we could confirm their MBB0. Univariate and multivariate logistic regression analyses were performed to examine whether MBB0 or BMI on admission was associated with advanced diabetic retinopathy or nephropathy. To evaluate the predictive performance of these indexes, we performed cross-validation in various models with $\mathrm{MBBO}$ or $\mathrm{BMI}$ and evaluated the areas under the curve (AUCs) yielded by these analyses.

Results Univariate analyses suggested that MBBO was associated with advanced retinopathy and nephropathy, while BMI on admission was associated only with advanced nephropathy. In multivariate analyses, MBBO was significantly associated with advanced complications, while BMI on admission was not. For advanced diabetic retinopathy, the AUCs were $0.70-0.72$, and for advanced nephropathy, the AUCs were $0.81-0.83$. When comparing the AUCs among models, the models with MBB0 sustained high predictive performance for diabetic complications. Conclusions MBBO was independently associated with advanced diabetic complications, while BMI on admission was not. Diabetic microvascular complications in patients with high MBBO could progress more rapidly. At the time of the diagnosis of diabetes mellitus, MBB0 would enable us to predict the progress of diabetic complications.

\section{INTRODUCTION}

Type 2 diabetes mellitus is a common disease with a high comorbidity rate worldwide. ${ }^{1}$ Diabetic complications deteriorate patients' quality of life and activities of daily living. ${ }^{23}$ Diabetic retinopathy (DR) develops at a relatively early stage. Untreated DR leads

\section{Significance of this study}

What is already known about this subject?

- The maximum body mass index before onset of type 2 diabetes (MBBO) might be used to estimate a patient's insulin secretion capacity.

- There have been few factors that can predict future diabetic complications at the time of diagnosis of diabetes mellitus.

What are the new findings?

- High MBBO is independently associated with advanced diabetic retinopathy.

- High MBBO is independently associated with advanced diabetic nephropathy.

- Body mass index on admission was not associated with advanced diabetic complications.

How might these results change the focus of research or clinical practice?

- At the time of the diagnosis of diabetes mellitus, MBBO would enable us to predict the progress of diabetic complications, and this prediction would lead to more careful support and medical treatment.

to a decrease in eyesight and ultimately blindness. ${ }^{3}$ Diabetic nephropathy (DN) is the most common disease that requires hemodialysis. ${ }^{4}$ Both of these complications seriously decrease patients' quality of life and activities of daily living unless treated appropriately. Prevention of DR or DN progression is one of the most important problems to be solved. Although some previous studies indicated that past obesity was associated with diabetic complications, these studies used past maximum body weight regardless of the onset of diabetes. ${ }^{5-7}$ Treatment with insulin, sulfonylureas or thiazolidinediones could increase body weight, ${ }^{8} 9$ while treatment with sodium-glucose cotransporter-2 inhibitors $^{10} 11$ or glucagon-like peptide-1 
receptor agonists ${ }^{12} 13$ could reduce body weight. Thus, the maximum body weight after the development of diabetes could be different from the maximum body weight before the onset of diabetes.

We reported that maximum body mass index before the onset of diabetes (MBBO) was independently correlated with insulin secretion and might be used to estimate a patient's insulin secretion capacity over the course of the disease. ${ }^{14}$ Previous studies reported the association between hyperinsulinemia and angiopathy. ${ }^{15} 16$ We assumed that patients with high MBBO who might have increased insulin levels could be susceptible to angiopathy. In addition, there have been few factors that can predict future diabetic complications at the time of diagnosis of diabetes mellitus. Thus, we focused on the relationship between $\mathrm{MBBO}$, as a factor that can be identified at diagnosis, and diabetic complications.

The purpose of this study was to assess the usefulness of $\mathrm{MBBO}$ as a predictor of progressive DR or DN in patients with type 2 diabetes mellitus. We enrolled patients with diabetes admitted to our hospital and analyzed the correlations between various clinical parameters, including $\mathrm{MBBO}$ and $\mathrm{DR}$ or $\mathrm{DN}$, in multiple regression analyses. We also included hypertension, dyslipidemia and hyperuricemia, which are associated with arteriosclerosis, in this analysis. ${ }^{1718}$

\section{RESEARCH DESIGN AND METHODS}

\section{Patients}

We retrospectively reviewed 1304 consecutive patients with type 2 diabetes who were admitted to Osaka University Hospital from August 2010 to June 2017 for treatment of poor glycemic control. Data for the present study were obtained from the medical records of Osaka University Hospital. The patient flow diagram is shown in figure 1. Fifty-eight subjects were excluded because their maximum body mass index (BMI) was not recorded; 298 subjects whose maximum BMI was reached after the diagnosis of type 2 diabetes mellitus were also excluded, as we could not identify their MBBO. Furthermore, the following patients were also excluded from the study; 187 patients with cancer, 51 patients with pancreatic diseases,

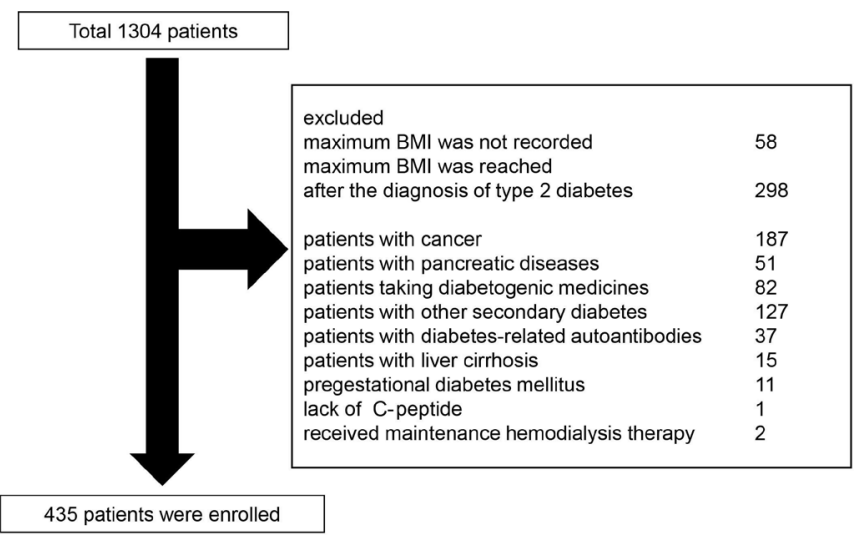

Figure 1 Patient flow diagram. BMI, body mass index.
15 patients with liver cirrhosis, 82 patients taking diabetogenic medicines such as glucocorticoids, 127 patients with an additional secondary form of diabetes including endocrine diseases such as pituitary, thyroid and adrenal diseases. Eleven patients who had pregestational diabetes mellitus, and 37 patients with diabetes-related autoantibodies were excluded. One patient was excluded due to the lack of C-peptide data, and two patients were excluded because they received maintenance hemodialysis therapy. Finally, 435 patients were enrolled in this study.

\section{Data collection}

After admission, most of the patients were treated with medical nutrition therapy plus bolus insulin therapy to improve preprandial plasma glucose levels, including fasting plasma glucose (FPG), the target level of which was below $8.4 \mathrm{mmol} / \mathrm{L}$. We performed a blood evaluation before breakfast, which was 12 hours after the last meal. Some of the patients were also treated with additional oral hypoglycemic agents or basal insulin. After glycemic control was maintained almost at the target levels, beta-cell function was evaluated. Beta-cell function was evaluated using the C-peptide index (CPI), which we previously reported to be correlated with pancreatic beta-cell mass and insulin secretory capacity. ${ }^{19}$ CPI was calculated by using the following formula: F-CPR (ng/ $\mathrm{mL}) \times 100 /$ FPG $(\mathrm{mmol} / \mathrm{L}) \times 18$. The medications used as glucose-lowering agents before admission and at evaluation are described in table 1 . We defined the onset of type 2 diabetes mellitus as the point when the patient had been diagnosed with type 2 diabetes mellitus based on the criteria of the American Diabetes Association ${ }^{20}$ or started taking glucose-lowering agents. We defined the patient's MBBO based on his or her history of maximum $\mathrm{BMI}$ and age at diabetes mellitus onset.

The period from the age at MBBO to admission was designated as $\Delta$ year. We investigated each patient's family history of type 2 diabetes in second-degree relatives, smoking history and current medication use. The severity of DR, which was diagnosed by an ophthalmologist, was assessed using modified Davis grading. Preproliferative diabetic retinopathy (prePDR) or more severe retinopathy was defined as advanced DR. Advanced DN was defined based on the eGFR and the presence of macroalbuminuria or proteinuria as follows: macroalbuminuria $>300 \mathrm{mg} / \mathrm{gCr}$, proteinuria $>0.5 \mathrm{~g} / \mathrm{gCr}$ or eGFR $<30 \mathrm{~mL} / \mathrm{min} / 1.73 \mathrm{~m}^{2}$. To evaluate the presence of diabetic neuropathy, the abbreviated diagnostic criteria proposed by the Diabetic Neuropathy Study Group in Japan were used. ${ }^{21}$ The criteria consisted of three items: (1) symptoms such as tingling pain, numbness and cramping; (2) absence of the Achilles tendon reflex; and (3) a reduced sense of vibration; neuropathy was diagnosed when more than two items were present. A history of cardiovascular disease (CVD) events was defined as a medical history of acute myocardial infarction, cerebral hemorrhage or infarction requiring hospitalization 
Table 1 Clinical characteristics of the study subjects

\begin{tabular}{|c|c|c|c|c|c|}
\hline MBBO & $\begin{array}{l}\text { All } \\
(n=435)\end{array}$ & $\begin{array}{l}\text { MBBO }<25 \\
(n=79)\end{array}$ & $\begin{array}{l}25 \leq M B B O<30 \\
(n=175)\end{array}$ & $\begin{array}{l}30 \leq \text { MBBO } \\
(n=181)\end{array}$ & $P$ value \\
\hline Age (years) & $63(51 \sim 72)$ & $67(61 \sim 74)$ & $65(58 \sim 74)$ & $56(45 \sim 67)$ & $<0.0001$ \\
\hline $\operatorname{Sex}(M / F)$ & $262 / 173$ & $48 / 31$ & $103 / 72$ & $111 / 70$ & 0.89 \\
\hline Age at diagnosis of T2DM (years) & $50(42 \sim 60)$ & $56(47 \sim 64)$ & $53(45 \sim 61)$ & $44(36 \sim 53)$ & $<0.0001$ \\
\hline Duration (years) & $10(2 \sim 18)$ & $10(2 \sim 19)$ & $11(2 \sim 19)$ & $10(3 \sim 18)$ & 0.92 \\
\hline BMI on admission $\left(\mathrm{kg} / \mathrm{m}^{2}\right)$ & $25.0(22.3 \sim 28.5)$ & $21.0(18.7 \sim 22.1)$ & $24.0(22.4 \sim 25.5)$ & $29.0(26.3 \sim 32.3)$ & $<0.0001$ \\
\hline $\operatorname{MBBO}\left(\mathrm{kg} / \mathrm{m}^{2}\right)$ & $28.8(25.8 \sim 33.1)$ & $23.2(21.8 \sim 24.3)$ & $27.1(26.2 \sim 28.6)$ & $34.1(31.6 \sim 38.0)$ & $<0.0001$ \\
\hline Age at MBBO (years) & $38(28 \sim 50)$ & $35(20 \sim 50)$ & $40(30 \sim 52)$ & $34(25 \sim 42)$ & $<0.0001$ \\
\hline$\Delta$ year (years) & $22(11 \sim 33)$ & $29(15 \sim 43)$ & $21(11 \sim 33)$ & 21 (10 31) & 0.0002 \\
\hline Family history & $237(55.0)$ & $40(50.6)$ & $98(56.6)$ & $99(55.3)$ & 0.67 \\
\hline Smoking history & $225(54.2)$ & $44(59.5)$ & $92(55.4)$ & $89(50.9)$ & 0.42 \\
\hline HbA1c (\%) & $8.5(7.8 \sim 9.9)$ & $8.2(7.5 \sim 9.9)$ & $8.4(7.7 \sim 9.7)$ & $8.8(8.0 \sim 10.5)$ & 0.0122 \\
\hline $\mathrm{HbA} 1 \mathrm{c}(\mathrm{mmol} / \mathrm{mol})$ & $70(62 \sim 85)$ & $66(58 \sim 85)$ & $68(61 \sim 83)$ & $73(64 \sim 91)$ & 0.0122 \\
\hline FPG (mmol/L) & $7.2(6.3 \sim 8.4)$ & $7.3(6.1 \sim 8.3)$ & $7.4(6.4 \sim 8.6)$ & $7.1(6.1 \sim 8.4)$ & 0.74 \\
\hline CPI & $1.1(0.76 \sim 1.7)$ & $0.79(0.48 \sim 1.1)$ & $1.1(0.78 \sim 1.6)$ & $1.3(0.86 \sim 2.1)$ & $<0.0001$ \\
\hline Advanced retinopathy & $116(26.8)$ & $16(20.3)$ & $38(21.8)$ & $62(34.4)$ & 0.0097 \\
\hline Advanced nephropathy & $72(16.6)$ & $7(8.9)$ & $19(10.9)$ & $46(25.4)$ & 0.0002 \\
\hline Neuropathy & $178(40.9)$ & $32(40.5)$ & $70(40.0)$ & $84(46.4)$ & 0.19 \\
\hline History of CVD & 91 (20.9) & $12(15.2)$ & $42(24.0)$ & $37(20.4)$ & 0.27 \\
\hline Hypertension & $251(57.7)$ & 35 (44.3) & $98(56.0)$ & $118(65.2)$ & 0.0062 \\
\hline Dyslipidemia & $258(59.3)$ & $39(49.4)$ & $113(64.6)$ & $106(58.6)$ & 0.071 \\
\hline Hyperuricemia & $101(23.3)$ & $12(15.2)$ & $36(20.6)$ & $63(34.8)$ & 0.024 \\
\hline \multicolumn{6}{|l|}{ Medication before admission } \\
\hline Sulfonylurea & $167(38.4)$ & $34(43.0)$ & $70(40.0)$ & $63(34.8)$ & 0.38 \\
\hline Glinide & $14(3.2)$ & $1(1.3)$ & $5(2.9)$ & $8(4.4)$ & 0.39 \\
\hline Biguanide & $103(23.7)$ & $11(13.9)$ & $34(19.4)$ & $58(32.0)$ & 0.0015 \\
\hline TZD & $35(8.0)$ & $5(6.3)$ & $13(7.4)$ & $17(9.4)$ & 0.65 \\
\hline$\alpha-G l$ & 75 (17.2) & $10(12.7)$ & $36(20.6)$ & $29(16.0)$ & 0.26 \\
\hline DPP-4i & $153(35.2)$ & $33(41.8)$ & $55(31.4)$ & $65(35.9)$ & 0.27 \\
\hline SGLT2i & $10(2.3)$ & $0(0)$ & $1(0.6)$ & $9(5.0)$ & 0.0069 \\
\hline GLP-1RA & $18(4.1)$ & $1(1.3)$ & $3(1.7)$ & $14(7.7)$ & 0.0063 \\
\hline Insulin & 117 (26.9) & 19 (24.1) & $44(25.1)$ & $54(29.8)$ & 0.50 \\
\hline No medication & $90(20.7)$ & $16(20.3)$ & 39 (22.3) & 35 (19.3) & 0.79 \\
\hline \multicolumn{6}{|l|}{ Medication at evaluation } \\
\hline Insulin secretagogues & $63(14.5)$ & $9(11.4)$ & $23(13.1)$ & $31(17.1)$ & 0.39 \\
\hline NPH or LAI & $195(44.8)$ & $31(39.2)$ & $65(37.1)$ & $99(54.7)$ & 0.0021 \\
\hline
\end{tabular}

Data are reported as the mean $\pm \mathrm{SD}$, median (IQR) or $\mathrm{n}(\%)$, unless otherwise indicated. Comparisons among the three groups divided by MBBO were performed by the Kruskal-Wallis test or the $\chi^{2}$ test for data presented as the median (IQR) or $\mathrm{n}(\%)$, respectively. $P$ values $<0.05$ were considered statistically significant.

The insulin secretagogues were sulfonylureas, glinides, DPP-4is and GLP-1RAs.

BMI, body mass index; CPI, C-peptide index; CVD, cardiovascular disease; DPP-4i, dipeptidyl peptidase-4 inhibitor; F, female; FPG, fasting plasma glucose; GLP-1RA, glucagon-like peptide-1 receptor antagonist; HbA1c, hemoglobin A1c; LAI, long-acting insulin; M, male; MBBO, maximum BMI before onset; NPH, neutral protamine Hagedorn; SGLT2i, sodiumglucose cotransporter 2 inhibitor; T2DM, type 2 diabetes mellitus; TZD, thiazolidinedione; $\alpha$-Gl, alpha-glucosidase inhibitor.

and angina pectoris requiring coronary revascularization treatment that included coronary intervention and coronary bypass graft. The presence of hypertension in patients was defined as systolic blood pressure $\geq 140 \mathrm{~mm}$ $\mathrm{Hg}$, diastolic blood pressure $\geq 90 \mathrm{~mm} \mathrm{Hg}$, or treatment with antihypertensive agents. The presence of 
dyslipidemia in patients was defined as LDL-C $\geq 140 \mathrm{mg}$ / $\mathrm{dL}$, triglycerides $\geq 150 \mathrm{mg} / \mathrm{dL}$, or treatment with lipidlowering agents. The presence of hyperuricemia was defined as uric acid $\geq 7.1 \mathrm{mg} / \mathrm{dL}$ or treatment with uric acid-lowering drugs.

The study was announced to the public on the website of our department at Osaka University Hospital, and all patients were allowed to participate or refuse to participate in the study.

\section{Statistics and analyses}

We summarized the background variables by median (interquartile range; IQR) for continuous variables and by counts with proportions for the categorical variables. We considered three groups based on the MBBO (low group: $\mathrm{MBBO}<25 \mathrm{~kg} / \mathrm{m}^{2}$, intermediate group: $25 \mathrm{~kg}$ / $\mathrm{m}^{2} \leq \mathrm{MBBO}<30 \mathrm{~kg} / \mathrm{m}^{2}$, high group: $30 \mathrm{~kg} / \mathrm{m}^{2} \leq \mathrm{MBBO}$ ), and the background variables were also presented as median (IQR) for the continuous variables and counts with proportions for the categorical variables by MBBO groups. This division was performed to fully understand the characteristics of the groups and the distribution of complications in the groups. The continuous and categorical variables were compared among the three MBBO groups by using the Kruskal-Wallis test and $\chi^{2}$ test, respectively.

Univariate analyses were performed by logistic regression models with advanced retinopathy and nephropathy as the outcome. The explanatory variables of these analyses were as follows: age, sex, duration of diabetes, family history of type 2 diabetes, smoking history, BMI on admission, MBBO, $\Delta$ year, hemoglobin A1c (HbAlc), CPI, and the presence of hypertension, dyslipidemia, and hyperuricemia. Multivariate analyses were also performed with advanced retinopathy and nephropathy as the outcome by logistic regression. In the multivariate analyses, to investigate the impact of $\mathrm{BMI}, \mathrm{MBBO}$, and age at $\mathrm{MBBO}$ on prediction, a prediction model was constructed by using a stepwise method with backward selection for all variables that excluded them, and we evaluated whether adding these factors to the model would improve prediction accuracy by cross-validation. Model 1 was defined as the logistic regression model with variables that were selected from among all variables except BMI, MBBO, and age at MBBO by the backward stepwise method with the criterion of $\mathrm{p}<0.2$. Models 2 and 3 were defined as the models adding BMI on admission and $\mathrm{MBBO}$, respectively, in addition to the variables used in model 1 . Since $\mathrm{MBBO}$ is a past factor in comparison with other factors, a model adjusting the period ' $\Delta$ year' in addition to the variables used in model 3 was also constructed as model 4. The performance of prediction for each model was evaluated by the areas under the curve (AUCs) calculated by tenfold cross-validation, and these were compared among models according to the previous studies. ${ }^{22} 23$ We selected the largest AUC as the best performance model. All results generated by the logistic regression model were summarized by the OR with its $95 \%$ CIs and the corresponding $\mathrm{p}$ values. The significance level in all analyses was $\mathrm{p}<0.05$, and all statistical analyses were performed with JMP Pro 13 (SAS Institute Inc).

\section{RESULTS}

\section{Clinical characteristics of the study subjects}

The clinical characteristics of the subjects are shown in table 1 . The median $\mathrm{HbAlc}$ was $8.5 \%(70 \mathrm{mmol} / \mathrm{mol})$, and most of the subjects had poor glycemic control, which was reflected by the need for hospital treatment. Patients with $\mathrm{MBBO} \geq 30 \mathrm{~kg} / \mathrm{m}^{2}$ were younger and had a younger age at diagnosis of T2DM than those in the other groups. In the three groups divided by MBBO, the duration of diabetes was not different, but a higher value of MBBO was associated with a higher value of CPI. Before admission, $38.4 \%$ of subjects were treated with sulfonylureas, $3.2 \%$ with glinides, $23.7 \%$ with biguanides, $8.0 \%$ with thiazolidinediones, $17.2 \%$ with $\alpha$-glucosidase inhibitors, $35.2 \%$ with dipeptidyl peptidase- 4 inhibitors (DPP-4is), $2.3 \%$ with sodium-glucose cotransporter 2 inhibitors, $4.1 \%$ with glucagon-like peptide-1 receptor agonists (GLP-1RAs) and 26.9\% with insulin; 20.7\% were receiving no medication (table 1). At laboratory evaluation, $14.5 \%$ of subjects were treated with insulin secretagogues, including sulfonylureas, glinides, DPP4is or GLP-1RAs, and $44.8 \%$ were receiving basal insulin (neutral protamine Hagedorn or long-acting insulin) (table 1).

There was a higher rate of comorbidities such as hypertension, hyperuricemia, advanced retinopathy and nephropathy in the $\mathrm{MBBO} \geq 30 \mathrm{~kg} / \mathrm{m}^{2}$ group than in the other groups. Univariate logistic regression suggested that MBBO was associated with advanced retinopathy, while BMI on admission was not. However, univariate logistic regression also suggested that $\mathrm{MBBO}$ and $\mathrm{BMI}$ on admission could be associated with advanced nephropathy (table 2). There was no difference in proportion of history of CVD among the three groups divided by MBBO (table 1).

\section{MBBO was independently associated with advanced retinopathy}

As a result of stepwise backward elimination for advanced retinopathy, age, duration, smoking history, CPI and the presence of hypertension and hyperuricemia were selected (table 3, model 1). Second, a multiple logistic regression adding 'BMI on admission' to the variables used in model 1 was performed (table 3, model 2). The $p$ value of the analysis including BMI on admission was 0.85 , indicating no significant association between BMI on admission and advanced retinopathy. Then, a multiple logistic regression adding MBBO to the variables used in model 1 was performed (table 3, model 3). The $\mathrm{p}$ value of the analysis including $\mathrm{MBBO}$ was 0.041 , indicating a significant association between MBBO and advanced retinopathy. Finally, a multiple logistic regression adding $\Delta$ year to the variables used in model 3 was 
Table 2 Results of univariate logistic regression for diabetic microvascular complications

\begin{tabular}{|c|c|c|c|c|c|c|}
\hline & \multicolumn{3}{|c|}{$\begin{array}{l}\text { Model A: advanced retinopathy as the dependent } \\
\text { variable }\end{array}$} & \multicolumn{3}{|c|}{$\begin{array}{l}\text { Model B: advanced nephropathy as the dependent } \\
\text { variable }\end{array}$} \\
\hline & OR & $95 \% \mathrm{Cl}$ & $P$ value & OR & $95 \% \mathrm{Cl}$ & $P$ value \\
\hline Age (years) & 0.99 & 0.98 to 1.01 & 0.71 & 0.99 & 0.98 to 1.02 & 0.79 \\
\hline Sex & 1.09 & 0.71 to 1.70 & 0.69 & 3.21 & 1.77 to 6.18 & $<0.0001$ \\
\hline Duration (years) & 1.06 & 1.03 to 1.08 & $<0.0001$ & 1.04 & 1.02 to 1.07 & 0.0003 \\
\hline Family history & 1.17 & 0.76 to 1.80 & 0.49 & 0.84 & 0.51 to 1.41 & 0.51 \\
\hline Smoking history & 0.73 & 0.47 to 1.13 & 0.15 & 1.23 & 0.73 to 2.08 & 0.44 \\
\hline BMI on admission $\left(\mathrm{kg} / \mathrm{m}^{2}\right)$ & 1.01 & 0.97 to 1.05 & 0.68 & 1.06 & 1.02 to 1.11 & 0.0088 \\
\hline $\operatorname{MBBO}\left(\mathrm{kg} / \mathrm{m}^{2}\right)$ & 1.05 & 1.01 to 1.08 & 0.013 & 1.10 & 1.06 to 1.15 & $<0.0001$ \\
\hline$\Delta$ year (years) & 1.03 & 1.01 to 1.04 & 0.0001 & 1.02 & 1.01 to 1.04 & 0.0047 \\
\hline $\mathrm{HbA1c}(\%)$ & 1.08 & 0.97 to 1.20 & 0.17 & 1.08 & 0.95 to 1.23 & 0.22 \\
\hline $\mathrm{CPI}$ & 0.72 & 0.53 to 0.95 & 0.019 & 1.58 & 1.21 to 2.08 & 0.0010 \\
\hline Hypertension & 2.03 & 1.30 to 3.22 & 0.0018 & 7.57 & 3.73 to 17.52 & $<0.0001$ \\
\hline Dyslipidemia & 1.17 & 0.76 to 1.81 & 0.49 & 1.70 & 1.00 to 2.98 & 0.0499 \\
\hline Hyperuricemia & 2.33 & 1.45 to 3.74 & 0.0005 & 6.15 & 3.59 to 10.62 & $<0.0001$ \\
\hline
\end{tabular}

$\mathrm{BMI}$, body mass index; CPI, C-peptide index; HbA1c, hemoglobin A1c; MBBO, maximum BMI before onset.

performed (table 3 , model 4). The p value of the analysis including $\mathrm{MBBO}$ was 0.033 , and the $\mathrm{p}$ value of the analysis including $\Delta$ year was 0.023 , indicating that there was also a significant association between $\Delta$ year and advanced retinopathy. In comparing each AUC among the four models yielded by cross-validation, the models including MBBO exhibited slightly increased predictive performance for diabetic complications, but the increase of the performance was not statistically significant $(\mathrm{p}=0.20$ or $\mathrm{p}=0.14$ ). These analyses in model A revealed that high MBBO was independently associated with advanced retinopathy after adjusting for other variables, although BMI on admission was not.

\section{MBBO was also independently associated with advanced nephropathy}

As a result of stepwise backward elimination for advanced nephropathy, sex, duration, HbA1c, CPI and the presence of hypertension and hyperuricemia were selected (table 4, model 1). In model 2 shown in table 4, the $\mathrm{p}$ value of the analysis including BMI on admission was 0.46 , indicating no significant association between BMI on admission and advanced nephropathy. In model 3, the $\mathrm{p}$ value of the analysis including $\mathrm{MBBO}$ was 0.0018 , indicating a significant association between $\mathrm{MBBO}$ and advanced nephropathy (table 4). In model 4, MBBO was

\begin{tabular}{|c|c|c|c|c|c|c|c|c|c|c|c|c|}
\hline & \multicolumn{3}{|c|}{ Model 1} & \multicolumn{3}{|c|}{ Model 2} & \multicolumn{3}{|c|}{ Model 3} & \multicolumn{3}{|c|}{ Model 4} \\
\hline & OR & $95 \% \mathrm{CI}$ & $P$ value & OR & $95 \% \mathrm{CI}$ & $P$ value & OR & $95 \% \mathrm{CI}$ & $P$ value & OR & $95 \% \mathrm{Cl}$ & $P$ value \\
\hline Age & 0.96 & 0.94 to 0.99 & 0.0018 & 0.97 & 0.94 to 0.99 & 0.0037 & 0.97 & 0.95 to 0.99 & 0.026 & 0.96 & 0.94 to 0.99 & 0.0050 \\
\hline Duration & 1.06 & 1.03 to 1.09 & $<0.0001$ & 1.06 & 1.03 to 1.09 & $<0.0001$ & 1.06 & 1.03 to 1.09 & $<0.0001$ & 1.05 & 1.02 to 1.08 & 0.0021 \\
\hline Smoking & 0.73 & 0.45 to 1.18 & 0.20 & 0.73 & 0.46 to 1.18 & 0.20 & 0.76 & 0.47 to 1.22 & 0.25 & 0.74 & 0.46 to 1.21 & 0.23 \\
\hline $\mathrm{HbA1c}$ & 1.09 & 0.96 to 1.25 & 0.18 & 1.10 & 0.96 to 1.25 & 0.18 & 1.08 & 0.94 to 1.24 & 0.25 & 1.09 & 0.95 to 1.25 & 0.21 \\
\hline $\mathrm{CPI}$ & 0.63 & 0.42 to 0.90 & 0.015 & 0.62 & 0.41 to 0.91 & 0.019 & 0.54 & 0.35 to 0.81 & 0.0036 & 0.56 & 0.36 to 0.83 & 0.0052 \\
\hline HT & 2.18 & 1.24 to 3.86 & 0.0072 & 2.15 & 1.20 to 3.87 & 0.011 & 1.91 & 1.07 to 3.42 & 0.030 & 1.85 & 1.03 to 3.33 & 0.040 \\
\hline HUA & 2.35 & 1.33 to 4.16 & 0.0032 & 2.35 & 1.33 to 4.16 & 0.0032 & 2.45 & 1.38 to 4.36 & 0.0023 & 2.38 & 1.34 to 4.25 & 0.0033 \\
\hline BMI & & & & 1.01 & 0.95 to 1.07 & 0.85 & & & & & & \\
\hline MBBO & & & & & & & 1.05 & 1.00 to 1.11 & 0.041 & 1.05 & 1.00 to 1.11 & 0.033 \\
\hline$\Delta$ year & & & & & & & & & & 1.03 & 1.00 to 1.05 & 0.023 \\
\hline & \multicolumn{3}{|c|}{ AUC: 0.704} & \multicolumn{3}{|c|}{ AUC: 0.701} & \multicolumn{3}{|c|}{ AUC: 0.715} & \multicolumn{3}{|c|}{ AUC: 0.723} \\
\hline
\end{tabular}

Model 1: all explanatory variables except BMI on admission, MBBO, and age at MBBO were included and then excluded if the variable was not statistically significant at a $20 \%$ level $(\mathrm{p}<0.20)$ via stepwise backward elimination. Model 2: model 1+BMI on admission; model 3: model 1+MBBO; model 4: model 3+ 4 year.

AUC, area under the curve; BMI, body mass index; CPI, C-peptide index; HbA1c, hemoglobin A1c; HT, hypertension; HUA, hyperuricemia; MBBO, maximum BMI before onset; $\triangle \mathrm{year}$, the period from age at MBBO to admission. 


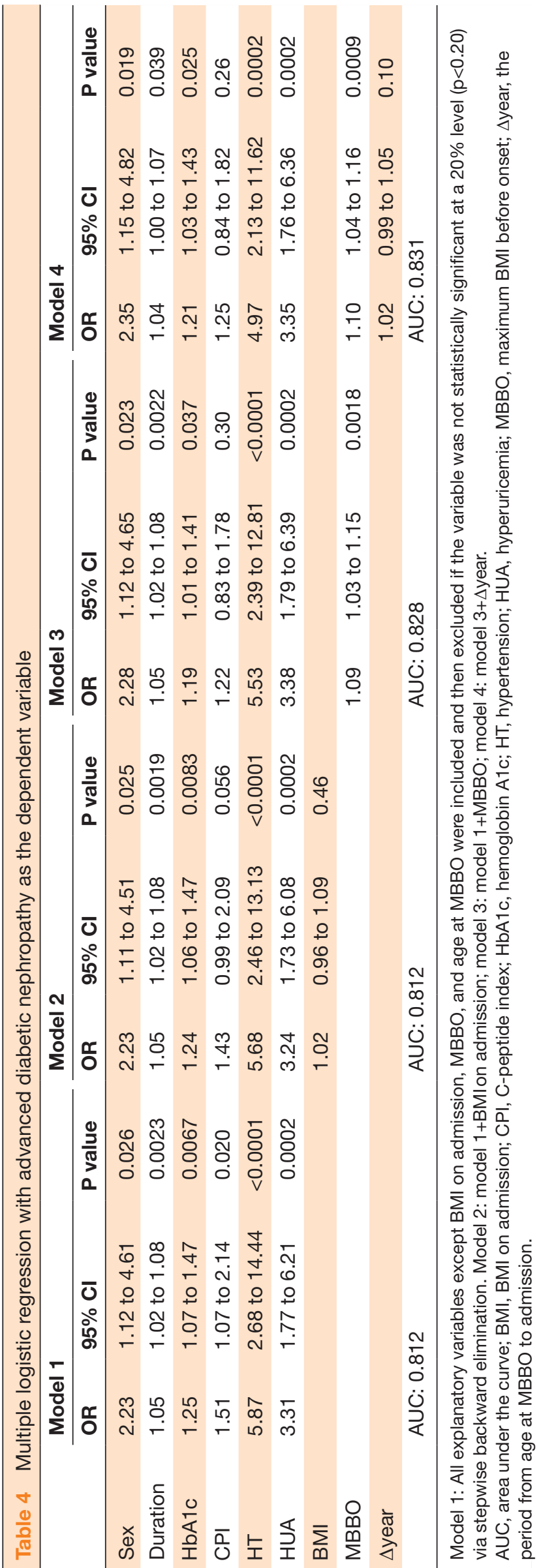

significant, but $\Delta y e a r$ was not significant ( $\mathrm{p}$ values of the analyses including MBBO and $\triangle$ year were 0.0009 and 0.10 , respectively), indicating that there was no significant association between $\Delta$ year and advanced nephropathy (table 4). In comparing each AUC among the four models yielded by cross-validation, the models including MBBO exhibited slightly increased predictive performance for diabetic complications, but the increase of the performance was not statistically significant $(\mathrm{p}=0.13$ or $\mathrm{p}=0.083$ ). These analyses for advanced nephropathy revealed that a high MBBO was also independently associated with advanced nephropathy after adjusting for other variables, although BMI on admission was not.

\section{CONCLUSIONS}

This study revealed that MBBO would enable us to predict the progress of patient's diabetic complications at the time of diagnosis of diabetes mellitus. We showed that MBBO was independently associated with advanced retinopathy and nephropathy, while BMI on admission was not. The risk factors previously reported for the development of DR include hyperglycemia, diabetes duration, hypertension, and $\mathrm{HbA1c}$, while among obesity-related factors, BMI ${ }^{24}$ past maximum BMI (regardless of onset of diabetes),${ }^{56}$ and metabolic syndrome were also reported.$^{25}$ The risk factors previously reported for the development of DN include obesity, hypertension, dyslipidemia, hyperuricemia, ${ }^{26}$ and $\mathrm{HbAlc},{ }^{27}$ while past maximum BMI (regardless of onset of diabetes) was also reported. ${ }^{6}$ As MBBO is the past maximum BMI of the subjects in this study, the results in this study are consistent with those of previous studies. However, little has been known as a factor forecasting diabetic complications at the time of diagnosis of diabetes mellitus. This study has the advantage of showing that MBBO would enable us to estimate the risk of advanced diabetic complications at the time of diagnosis of diabetes mellitus.

This study revealed the association between $\mathrm{MBBO}$ and advanced complications. As we previously reported that high MBBO was associated with high insulin secretion levels, ${ }^{14}$ patients with high MBBO may present hyperinsulinemia due to insulin resistance related to obesity. A previous study showed that hyperinsulinemia induced inflammatory processes in the arteries of rats despite normal glucose levels. ${ }^{15}$ In vascular smooth muscle cells of hyperinsulinemic rats, insulin increased the levels of inflammatory cytokines, which were reported to be aggravating factors for retinopathy ${ }^{28}$ and nephropathy. ${ }^{29}$ Thus, hyperinsulinemia could be one of the possible mechanisms underlying the association between MBBO and retinopathy or nephropathy. Another possible mechanism might be associated with abnormal adipocytokine secretion, which is derived from obesity ${ }^{30}$ and could affect microvascular complications. ${ }^{31}$ The high MBBO group might have been affected by hyperinsulinemia and obesity, perhaps for a long time, even before the onset of diabetes. While the progression of diabetic complications 
is affected by glycemic control and diabetes duration, MBBO, which allows us to estimate a patient's risk of diabetic complications at the time of diagnosis of diabetes mellitus, could be a superior factor for predicting the development of diabetic complications.

BMI at entry has been used in most studies that examine the relationship between body weight and disease. In our study, we used MBBO or BMI on admission in multiple logistic regression analyses. In multiple logistic regression, there was no significant association between BMI on admission and advanced retinopathy or nephropathy. To clarify the characteristics of the BMI groups on admission, we divided patients into two groups based on BMI on admission (normal BMI group: BMI on admission $<25 \mathrm{~kg} / \mathrm{m}^{2}, \mathrm{n}=220$; high BMI group: BMI on admission $\geq 25 \mathrm{~kg} / \mathrm{m}^{2}, \mathrm{n}=215$ ), and continuous and categorical variables were compared between the two BMI groups on admission by using the Kruskal-Wallis test and $\chi^{2}$ test, respectively. This comparison revealed that subjects in the high BMI group had more advanced diabetic microvascular complications than those in the normal BMI group (normal BMI group vs high BMI group; retinopathy: $23.3 \%$ vs $30.4 \%, \mathrm{p}=0.096$; nephropathy: $11.9 \%$ vs $21.4 \%, \mathrm{p}=0.0077$ ), while the high BMI group had a shorter diabetes duration than the normal BMI group (7 vs 13 years, $\mathrm{p}<0.0001)$. Thus, the high BMI group could have an earlier onset of advanced diabetic microvascular complications than the normal BMI group. These results indicate the importance of treatment for current obesity to control diabetic complications. Considering that BMI does not indicate the existence or duration of past hyperinsulinemia, we should not overlook the high risk for diabetic complications in patients with normal BW at present.

Additionally, we analyzed the contribution of $\triangle \mathrm{BMI}$ (MBBO minus BMI on admission) to advanced diabetic microvascular complications. We divided the patients into two groups based on their $\triangle \mathrm{BMI}$ and compared the continuous and categorical variables between the two $\Delta$ BMI groups by using the Kruskal-Wallis test and $\chi^{2}$ test, respectively. As a result, more patients had advanced diabetic complications in the higher $\Delta \mathrm{BMI}$ group. There was no difference in BMI on admission between these two groups, while patients with higher $\Delta \mathrm{BMI}$ had younger ages of diabetes diagnosis, longer duration of diabetes and higher MBBO, suggesting that they could have been exposed to higher serum insulin level in the longer duration of obesity, resulting in the advanced diabetic complications.

In this study, we also analyzed the association of DR and DN with hypertension, dyslipidemia and hyperuricemia, which are well-known causes of arteriosclerosis. ${ }^{32-34}$ We found that hypertension or hyperuricemia was independently associated with advanced complications, and these findings are consistent with previous research. ${ }^{24} 263536$ However, dyslipidemia was not associated with advanced complications. A recent study demonstrated that dyslipidemia was involved in the development of $\mathrm{DR} .{ }^{37}$ As we did not examine the relationship between actual cholesterol level and diabetic complications, the relationship between dyslipidemia and diabetic complications should be examined using the cholesterol level after adjusting medication for dyslipidemia.

This study had some limitations. First, it was a retrospective cross-sectional observational study. Prospective longitudinal studies evaluating the effects of treatments are necessary to clarify the actual change in individual patient complications. Second, we excluded many patients whose maximum BMI was reached after the diagnosis of type 2 diabetes mellitus from this study. BMI that reached a maximum level after the development of type 2 diabetes mellitus was affected by the use of hypoglycemic agents and/or insulin and would not indicate the patients' potential body weight changes. Third, we did not evaluate medications for hypertension, dyslipidemia or hyperuricemia. It would be necessary for more accurate analyses to adjust for these medications. Finally, maximum insulin secretion can be observed before the onset of diabetes mellitus in Japanese patients with type 2 diabetes. However, maximum insulin secretion may be observed even after the onset of diabetes mellitus in other populations. Therefore, the observation shown in this study may only be true for the Japanese patients.

In conclusion, MBBO was independently associated with advanced diabetic complications. Diabetic microvascular complications in patients with high MBBO could progress more rapidly. Therefore, we need to consider complications in these patients more carefully.

Acknowledgements We would like to thank our colleagues from Osaka University Center of Medical Data Science and Advanced Clinical Epidemiology Investigator's Research Project for providing their insight and expertise in our research.

Contributors $\mathrm{HO}, \mathrm{KF}$ and $\mathrm{HI}$ designed the whole project and wrote the manuscript. $\mathrm{HO}$ contributed to the acquisition and analysis of the data. SF and YF assisted with the data analysis and reviewed and edited the manuscript. TK and JK assisted with the study design and reviewed and edited the manuscript. AT reviewed and edited the manuscript. SK provided statistical advice regarding the study design and assisted with the data analysis. IS assisted with the study design and analysis and reviewed and edited the manuscript. All authors revised the manuscript critically for important intellectual content and approved the final version of the manuscript. $\mathrm{KF}$ is the guarantor of this work.

Funding The authors have not declared a specific grant for this research from any funding agency in the public, commercial or not-for-profit sectors.

Competing interests None declared.

Patient consent for publication Not applicable.

Ethics approval This study was approved by the Institutional Ethics Review Board of Osaka University Hospital (approval number: 16136-3) and was carried out in accordance with the principles of the Declaration of Helsinki

Provenance and peer review Not commissioned; externally peer reviewed.

Data availability statement All data relevant to the study are included in the article. The datasets generated and analyzed during the current study are available from the corresponding author on reasonable request.

Open access This is an open access article distributed in accordance with the Creative Commons Attribution Non Commercial (CC BY-NC 4.0) license, which permits others to distribute, remix, adapt, build upon this work noncommercially, and license their derivative works on different terms, provided the original work is properly cited, appropriate credit is given, any changes made indicated, and the use is non-commercial. See: http://creativecommons.org/ licenses/by-nc/4.0/. 
ORCID iDs

Harutoshi Ozawa http://orcid.org/0000-0001-7590-6745

Kenji Fukui http://orcid.org/0000-0003-2468-7559

Yukari Fujita http://orcid.org/0000-0002-8847-5654

Junji Kozawa http://orcid.org/0000-0001-9665-8947

\section{REFERENCES}

1 Saeedi P, Salpea P, Karuranga S, et al. Mortality attributable to diabetes in 20-79 years old adults, 2019 estimates: Results from the International Diabetes Federation Diabetes Atlas, 9th edition. Diabetes Res Clin Pract 2020;162:108086.

2 Haneda M, Noda M, Origasa $\mathrm{H}$. Japanese clinical practice guideline for diabetes 2016. Diabetol Int 2018;27:1-45.

3 Chung WK, Erion K, Florez JC, et al. Precision medicine in diabetes: a consensus report from the American diabetes association (ADA) and the European association for the study of diabetes (EASD). Diabetes Care 2020;43:1617-35.

4 Masakane I, Taniguchi M, Nakai S, et al. Annual dialysis data report 2016, JSDT renal data registry. Renal Replacement Therapy 2018:4:1-45.

5 Ogawa K-ichi, Ueda K, Sasaki H, et al. History of obesity as a risk factor for both carotid atherosclerosis and microangiopathy. Diabetes Res Clin Pract 2004;66:S165-8.

6 Tanaka S, Tanaka S, limuro S, et al. Maximum BMI and microvascular complications in a cohort of Japanese patients with type 2 diabetes: the Japan diabetes complications study. J Diabetes Complications 2016;30:790-7.

7 Meguro S, Kabeya Y, Tanaka K, et al. Past obesity as well as present body weight status is a risk factor for diabetic nephropathy. Int $J$ Endocrinol 2013;2013:1-5.

8 Turner RC, Holman RR, Stratton IM. Effect of intensive bloodglucose control with metformin on complications in overweight patients with type 2 diabetes (UKPDS 34). Lancet 1998;352:854-65.

9 Dormandy JA, Charbonnel B, Eckland DJA, et al. Secondary prevention of macroVascular events in patients with type 2 diabetes in the proactive study (PROspective pioglitAzone clinical trial in macroVascular events): a randomised controlled trial. Lancet 2005;366:1279-89.

10 Zinman B, Wanner C, Lachin JM, et al. Empagliflozin, cardiovascular outcomes, and mortality in type 2 diabetes. $N$ Engl J Med 2015;373:2117-28.

11 Neal B, Perkovic V, Mahaffey KW, et al. Canagliflozin and cardiovascular and renal events in type 2 diabetes. N Engl J Med 2017;377:644-57.

12 Mann JFE, Fonseca V, Mosenzon O, et al. Effects of liraglutide versus placebo on cardiovascular events in patients with type 2 diabetes mellitus and chronic kidney disease. Circulation 2018;138:2908-18.

13 Gerstein HC, Colhoun HM, Dagenais GR, et al. Dulaglutide and cardiovascular outcomes in type 2 diabetes (REWIND): a doubleblind, randomised placebo-controlled trial. Lancet 2019;394:121-30.

14 Ozawa H, Fukui K, Komukai S, et al. Clinical significance of the maximum body mass index before onset of type 2 diabetes for predicting beta-cell function. J Endocr Soc 2020;4:bvz023.

15 Sekine O, Nishio Y, Egawa K, et al. Insulin activates CCAAT/ enhancer binding proteins and proinflammatory gene expression through the phosphatidylinositol 3-kinase pathway in vascular smooth muscle cells. J Biol Chem 2002;277:36631-9.

16 Sato $Y$, Nishio $Y$, Sekine O, et al. Increased expression of CCAAT/enhancer binding protein-beta and -delta and monocyte chemoattractant protein-1 genes in aortas from hyperinsulinaemic rats. Diabetologia 2007;50:481-9.

17 Vlajinac HD, Marinković JM, Maksimović MZ, et al. The prevalence of polyvascular disease in patients with carotid artery disease and peripheral artery disease. Kardiol Pol 2019;77:926-34.
18 Liang L, Hou X, Bainey KR, et al. The association between hyperuricemia and coronary artery calcification development: a systematic review and meta-analysis. Clin Cardiol 2019;42:1079-86.

19 Fujita Y, Kozawa J, Iwahashi H, et al. Increment of serum C-peptide measured by glucagon test closely correlates with human relative beta-cell area. Endocr J 2015;62:329-37.

20 American Diabetes Association. Classification and Diagnosis of Diabetes: Standards of Medical Care in Diabetes-2018. Diabetes Care 2018;41:S13-27.

21 Yasuda H, Sanada M, Kitada K, et al. Rationale and usefulness of newly devised abbreviated diagnostic criteria and staging for diabetic polyneuropathy. Diabetes Res Clin Pract 2007;77:S178-83.

22 Li P, Wang X, Xu C, et al. ${ }^{18}$ F-FDG PET/CT radiomic predictors of pathologic complete response ( $\mathrm{pCR}$ ) to neoadjuvant chemotherapy in breast cancer patients. Eur J Nucl Med Mol Imaging 2020;47:1116-26.

23 Keshavarz Z, Rezaee R, Nasiri M, et al. Obstructive sleep apnea: a prediction model using supervised machine learning method. Stud Health Technol Inform 2020;272:387-90.

24 Kawasaki R, Tanaka S, Tanaka S, et al. Incidence and progression of diabetic retinopathy in Japanese adults with type 2 diabetes: 8 year follow-up study of the Japan diabetes complications study (JDCS). Diabetologia 2011;54:2288-94.

25 Metascreen Writing Committee, Bonadonna RC, Cucinotta D, et al. The metabolic syndrome is a risk indicator of microvascular and macrovascular complications in diabetes: results from Metascreen, a multicenter diabetes clinic-based survey. Diabetes Care 2006;29:2701-7.

26 Molitch ME, Adler Al, Flyvbjerg A, et al. Diabetic kidney disease: a clinical update from kidney disease: improving global outcomes. Kidney Int 2015;87:20-30.

27 Altemtam N, Russell J, El Nahas M. A study of the natural history of diabetic kidney disease (DKD). Nephrol Dial Transplant 2012;27:1847-54.

28 Hammes H-P. Diabetic retinopathy: hyperglycaemia, oxidative stress and beyond. Diabetologia 2018;61:29-38.

29 John $\mathrm{P}$, Yadla M. Noninvasive method of differentiating diabetic nephropathy and nondiabetic renal disease using serum bone morphogenetic protein-7 and transforming growth factor-beta 1 levels in patients with type-2 diabetes mellitus. Saudi J Kidney Dis Transp/ 2019;30:1300-9.

30 Jung UJ, Choi M-S. Obesity and its metabolic complications: the role of adipokines and the relationship between obesity, inflammation, insulin resistance, dyslipidemia and nonalcoholic fatty liver disease. Int J Mol Sci 2014;15:6184-223.

31 Doganay S, Evereklioglu C, Er H, et al. Comparison of serum NO, TNF-alpha, IL-1beta, sIL-2R, IL-6 and IL-8 levels with grades of retinopathy in patients with diabetes mellitus. Eye 2002;16:163-70.

32 Wolde HF, Atsedeweyen A, Jember A, et al. Predictors of vascular complications among type 2 diabetes mellitus patients at University of Gondar referral Hospital: a retrospective follow-up study. BMC Endocr Disord 2018;18:52.

33 Sindhu S, Thomas R, Shihab $\mathrm{P}$, et al. Obesity is a positive modulator of IL-6R and IL-6 expression in the subcutaneous adipose tissue: significance for metabolic inflammation. PLoS One 2015;10:e0133494.

34 Lee J-J, Wang P-W, Yang I-H, et al. High-Fat diet induces Toll-like receptor 4-dependent macrophage/microglial cell activation and retinal impairment. Invest Ophthalmol Vis Sci 2015;56:3041-50.

35 Cui J, Ren J-P, Chen D-N, et al. Prevalence and associated factors of diabetic retinopathy in Beijing, China: a cross-sectional study. BMJ Open 2017;7:e015473.

36 Thounaojam MC, Montemari A, Powell FL, et al. Monosodium urate contributes to retinal inflammation and progression of diabetic retinopathy. Diabetes 2019;68:1014-25.

37 Jeng C-J, Hsieh Y-T, Yang C-M, et al. Diabetic retinopathy in patients with dyslipidemia: development and progression. Ophthalmol Retina 2018;2:38-45. 